\title{
Fertility restoration of Chinese wild rice-type cytoplasmic male sterility by CRISPR/Cas9-mediated genome editing of nuclear-encoded RETROGRADE-REGULATED MALE STERILITY
}

\author{
Chihiro Suketomo, Tomohiko Kazama a, Kinya Toriyama* \\ Graduate School of Agricultural Science, Tohoku University, 468-1 Aramaki Aza Aoba, Aoba-ku, Sendai, \\ Miyagi 980-8572, Japan \\ *E-mail: torikin@tohoku.ac.jp Tel: +81-22-757-4231 Fax: +81-22-757-4232
}

Received February 10, 2020; accepted March 26, 2020 (Edited by H. Shimada)

\begin{abstract}
Cytoplasmic male sterility (CMS) is a trait that produces nonfunctional pollen caused by the interaction between mitochondrial and nuclear genes. In Chinese-wild (CW) type CMS, CWA, in rice (Oryza sativa L.), its mitochondria enhance the expression of the nuclear gene RETROGRADE-REGULATED MALE STERILITY (RMS), which causes pollen abortion. Fertility is recovered when its expression decreases in a restorer line, CWR. The expression of RMS is controlled by the single nucleotide polymorphism (SNP) located in the promoter region 2,286 bp upstream of the start codon of $R M S$. However, another gene, PPR2, which encodes pentatricopeptide repeat-domain containing protein, is predicted in the reverse strand of this region and a premature stop codon is created in CWR by the SNP. To prove RMS is directly involved in restoring fertility of CW-CMS, we introduced mutations into RMS and PPR2 using CRISPR/Cas9. Fertility was recovered in the genome-edited CMS plants with reduced expression of RMS and unaltered expression of PPR2, when the mutation was introduced in the promoter regions of $R M S$ within or outside the coding sequence (CDS) of PPR2. Fertility restoration was not obtained when the mutation was introduced within the CDS of RMS. Our results demonstrated that PPR2 is not responsible for fertility restoration, and fertility was recovered by reduced expression of $R M S$, providing us with a new artificial fertility restorer line for agronomical use.
\end{abstract}

Key words: CRISPR-Cas9, cytoplasmic male sterility, fertility restoration, genome editing, Oryza sativa.

\section{Introduction}

Cytoplasmic male sterility (CMS), which is a maternally inherited trait resulting in the failure to produce functional pollen, is caused by the interaction between mitochondrial and nuclear genomes. Expression of a certain mitochondrial chimeric open reading frame (orf) gene, which is created through the recombination between different mitochondrial coding regions or between a mitochondrial gene and an unknown sequence, is known to have the ability to cause dysfunctions in pollen development and these orfs are termed as CMS-associated genes (Hanson and Bentolila 2004 for a review). In contrast, the expression of the CMS-associated orf is often suppressed by the expression of a particular nuclear-encoded gene, called the RESTORER OF FERTILITY $(R F)$ gene that rescues pollen development resulting in the recovery of fertility.
A CMS line is, in general, bred as a result of nuclear substitution to remove the functional $R F$ gene and allow the expression of a CMS-associated mitochondrial gene. A CMS and fertility restoration system, therefore, is an ideal trait to investigate the interaction between mitochondrial and nuclear genes.

Molecular cloning of $R F$ genes has been reported for maize (Zea mays L.), petunia (Petunia hybrida L.), rape (Brassica napus L.), sugar beet (Beta vulgaris L.), sorghum (Sorghum bicolor L.), and rice (Oryza sativa L.) (Bohra et al. 2016 for a review). The majority of $R F$ genes reported so far encode pentatricopeptide repeat (PPR) family proteins, which are known to be RNAbinding factors involved in processing or translation, or both, of organellar mRNAs (Schmitz-Linneweber and Small 2008). For example, in rice, Rf1a and Rf1b in Boro-Taichung (BT)-type CMS, Rf5 and Rf6 in Honglian (HL)-type CMS, and Rf4 in wild-abortive (WA)-type

Abbreviations: Cas9, CRISPR-associated endonuclease 9; CMS, cytoplasmic male sterility; CRISPR, clustered regularly interspaced short palindromic repeat; CDS, coding sequence; HPT, hygromycin phosphotransferase; PAM, protospacer-adjacent motif; PPR, pentatricopeptide repeat; RF, restorer of fertility; RT-PCR, reverse transcriptase-PCR; sgRNA, single-guide RNA; SNP, single nucleotide polymorphism; T65, Taichung 65.

a Present address: Faculty of Agriculture, Kyushu University, 744 Motooka, Nishi-ku, Fukuoka, Fukuoka 819-0385, Japan

This article can be found at http://www.jspcmb.jp/

Published online September 17, 2020 
CMS have been reported to encode PPR proteins and to function post-transcriptionally leading to the cleavage or degradation of mitochondrial transcripts of a CMSassociated gene (Akagi et al. 2004; Hu et al. 2012; Huang et al. 2015; Kazama et al. 2008; Kazama and Toriyama 2003, 2014; Komori et al. 2004; Tang et al. 2014; Wang et al. 2006). Some other $R F$ genes, such as $R f o$ in OguraCMS rape and $R f k 1$ in Kosena-CMS radish have been reported to regulate translation of mRNA of a CMSassociated gene (Koizuka et al. 2003; Uyttewaal et al. 2008).

In contrast, several $R F$ genes have been reported to encode non-PPR proteins. For example, $R f 2$ in maize encodes an aldehyde dehydrogenase (Liu and Schnable 2002), $R f 2$ in rice encodes a glycine-rich protein (Itabashi et al. 2011), bvORF20 in sugar beets encodes a protein resembling yeast OMA1, a peptidase involved in quality control of mitochondrial membrane proteins (Kitazaki et al. 2015), Rf4 in maize encodes a basic helix-loophelix (bHLH) transcription factor (Jaqueth et al. 2020), and $R f 17$, which is the $R F$ gene we investigated in the current study, for Chinese wild rice (CW)-type CMS in rice encodes an unknown protein with a segment similar to that of a part of an acyl carrier protein synthase-like domain (Fujii and Toriyama 2009).

The CW-type CMS is derived from Oryza rufipogon Griff. accession W1 (Fujii and Toriyama 2005). A CMS line, CWA, carries the cytoplasm of W1 and a nuclear genome of a Japonica Group cultivar Taichung 65 (T65) (Oryza sativa L.). A CMS-associated gene in CW-CMS has been reported to be a unique orf gene called CWorf307 (Fujii et al. 2010). The pollen grains of CWA are filled with starch and morphologically normal but lack germination ability, resulting in no seed setting. Its fertility is fully restored in the isonuclear restorer line CWR, which carries a single nuclear gene, $R f 17$ (Fujii and Toriyama 2005). Because all the $\mathrm{F}_{2}$ plants derived from the $\mathrm{F}_{1}$ hybrid between $\mathrm{CWA} \times \mathrm{CWR}$ are fertile, $R f 17$ behaves in a gametophytic manner. Molecular cloning of the $R f 17$ gene has revealed that $R f 17$ has reduced expression allele of RETROGRADE-REGULATED MALE STERILITY (RMS) (Fujii and Toriyama 2009; DDBJ accession number AB481199). The expression of the RMS allele (=rf17 allele) in the nucleus of the CMS line CWA is considered to be highly enhanced by a certain retrograde signal from the $\mathrm{CW}$ mitochondria, whereas the $r m s$ allele ( $=R f 17$ allele) in the fertility restorer line CWR might not percept the retrograde signal resulting in down-regulation of RMS expression and thus, restore fertility. The fertility restoration mechanism is quite distinct from that governed by PPR proteins. To the best of our knowledge, this type of fertility restoration has not been reported elsewhere.

The expression of RMS is known to be controlled by the single nucleotide polymorphism (SNP) located in the promoter region 2,286 bp upstream of the start codon of $R M S$, namely $R f 17$, which carries thymine, whereas $r f 17$ carries adenine (Fujii and Toriyama 2009). The other sequences, including the promoter region and coding sequence (CDS) are completely identical. However, we must note the SNP in CWR creates a premature stop codon in another gene (LOC_Os04g40010) encoding a PPR-domain containing protein, which was predicted in the reverse strand by the Michigan State University (MSU) Rice Genome Annotation Project Release 7. During map-based cloning of our previous study (Fujii and Toriyama 2009), we employed an RNAi approach and demonstrated that down-regulation of RMS (named ORF11 in the previous study) recovered fertility, whereas down-regulation of the $P P R$ gene (named $P P R 2$ in the previous and current studies) did not, leading to our proposal that PPR2 is not involved in fertility restoration. However, the RNAi-mediated suppression of RMS did not achieve the full restoration of seed setting. We were unable to find any mutants of PPR2 or RMS to date to rule out the possibility of involvement of PPR2 for fertility restoration and to prove the direct involvement of RMS in fertility restoration of CW-CMS.

In the present study, we employed a genomeediting technique, clustered regularly interspaced short palindromic repeats/CRISPR-associated protein 9 (CRISPR/Cas9), to knock out RMS and PPR2. The CRISPR/Cas9 system was developed in 2013 (Cong et al. 2013; Jiang et al. 2013; Shan et al. 2013) and effectively applied to plants to generate targeted mutations ( $\mathrm{Li}$ et al. 2013; Nekrasov et al. 2013). Mutations were introduced in the promoter of RMS within the CDS of PPR2, in the promoter of RMS outside the CDS of $P P R 2$, and inside the CDS of RMS. Investigation of fertility restoration and expression analysis of $R M S$ and $P P R 2$ revealed that $P P R 2$ was not related to fertility restoration of CW-CMS and reduced expression level of RMS was responsible for fertility restoration of CW-CMS.

\section{Materials and methods}

\section{Plant materials}

A CW-CMS line CWA and a restorer line CWR are isogenic lines and carry cytoplasm derived from Oryza rufipogon Griff. W1 strain, and nucleus of the Japonica Group cultivar Taichung 65 (T65). CWA carries $r f 17 r f 17$, whereas CWR carries Rf17Rf17 (Fujii and Toriyama 2005, 2009).

\section{Generation of genome-edited lines by CRISPR/ Cas9}

The Cas9 plant expression vector (pZH_gYSA_MMCas9) and sgRNA expression vector (pU6gRNA) were described by Mikami et al. (2015). According to the design principles of the target sequences in the CRISPR/Cas9 system, $20 \mathrm{nt}$ upstream of the PAM motif were selected as candidate target sequences 
using CRISPR direct (https://crispr.dbcls.jp/). Two different target sequences in the promoter and the coding region of RMS are listed in Supplementary Table S1. Double stranded target sequences, which were made by annealing the paired single oligonucleotides at $95^{\circ} \mathrm{C}$ for $5 \mathrm{~min}$, were cloned into the pU6gRNA vector, which was linearized by $\mathrm{Bbs}$ I. OsU3-gYSA in pZH_gYSA_MMCas9 was replaced by sgRNA expression constructs using Asc I and Pac I sites. The derived construct was introduced into the calli of CWA and T65 by Agrobacteriummediated transformation. The presence of the introduced sgRNA expression constructs was confirmed by detecting the gene for hygromycin phosphotransferase (HPT) using PCR. Primers are listed in Supplementary Table S2.

\section{Analysis of edited sequences}

To analyze the nucleotide sequences of the resultant mutants, genomic DNA was extracted from leaves of transgenic plants. PCR amplifications were conducted using primer pairs flanking the designed target sites, and are listed in Supplementary Table S3. IndelCheck ${ }^{\mathrm{TM}}$ CRISPR/TALEN Insertion or Deletion Detection System (GeneCopoeia, Rockville, MD, USA) were conducted according to the manufacturer's instructions. The nucleotide sequences were determined by direct sequencing of the PCR products or by sequencing of plasmids from at least two colonies after cloning of the PCR products into the pGEM-T-Easy-vector (Promega, WI, USA) using the CEQ8000 genetic analysis system (Beckman Coulter, https://www. beckmancoulter.com/). Genome-edited lines were selected and used in the following phenotypic analysis.

\section{Phenotypic analysis}

Plants were grown in a greenhouse at $30 / 25^{\circ} \mathrm{C}$ (day/night) under natural photoperiodic conditions or in a biotron $(\mathrm{LPH}-$ 410SPC; Nippon Medical \& Chemical Instruments Co., Ltd., Osaka, Japan $)$ at $30 / 25^{\circ} \mathrm{C}(10 \mathrm{~h}$ day/ $14 \mathrm{~h}$ night $)$, in which illumination was set at an intensity of $150 \mu \mathrm{mol}$ photons $\mathrm{m}^{-2} \mathrm{~s}^{-1}$ and a $\mathrm{CO}_{2}$ level was set at $500 \mathrm{ppm}$ (Ohnishi et al. 2011). Seed setting rates were determined by counting filled grains and total grains for at least three bagged panicles.

\section{qRT-PCR}

Total RNA from anthers at the tricellular pollen stages were extracted using RNeasy (Qiagen, Tokyo, Japan) according to the manufacturer's instructions. DNA contamination was eliminated by treatment with RNase-free DNase I (TaKaRa Bio Inc., Ohtsu, Japan). cDNA synthesis was accomplished using the ReverTraAce (TOYOBO, Osaka, Japan). PCR was performed for four biological replicates using TB Green Premix Ex Taq II (TaKaRa Bio Inc.) for quantitative RT-PCR analysis. Primers used for quantitative RT-PCR of Tubulin, RMS, and PPR2 are listed in Supplementary Table S4. Tukey's test was applied to test for differences among treatments at $p<0.05$ using the R package "multcomp” (Hothorn et al. 2008).

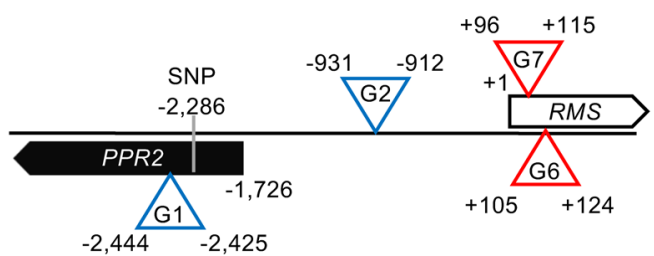

Figure 1. Target sequences on RMS and PPR2. Blue and red arrowheads indicate target sites on the promoter region of $R M S$ and on the CDS of RMS, respectively. The start codon of RMS is designated as +1 .

\section{Results}

Selection of CRISPR/Cas9 target sequences

To exclude the possibility that PPR2 is involved in the fertility restoration of CW-CMS, the target site G1 was designed within the PPR2 CDS and the target site $\mathrm{G} 2$ was in the promoter region, which resided between the RMS CDS and the PPR2 CDS (Figure 1). The target sites, G6 and G7, were selected within RMS CDS, and our goal was to directly prove the involvement of $R M S$ in fertility restoration of CW-CMS by knocking out the RMS (Figure 1). A synthetic $20 \mathrm{bp}$ long nucleotide corresponding to each target sequence was inserted into the binary vector cassette expressing a single guide RNA (sgRNA) and Cas9 endonuclease. We named these constructs G1, G2, G6, and G7, respectively. Each expression vector was introduced into the calli of the CMS line, CWA, and a maintainer line, Taichung T65 (T65) using the Agrobacterium-mediated transformation method.

\section{Sequence analysis of transgenic plants with $G 1$ and $\mathrm{G} 2$ constructs}

We generated 14 independent transgenic CWA plants with the G1 construct and seven with the G2 construct. The presence of the introduced foreign gene was confirmed by detection of the HPT gene using PCR (Supplementary Figure S1). The regenerated plants $\left(\mathrm{T}_{0}\right.$ plants) were screened for mutations using IndelCheck ${ }^{\mathrm{TM}}$ CRISPR/TALEN Insertion or Deletion Detection System. Four plants, named CWA_G1_a, CWA_G1_b, CWA_G1_c, and CWA_G1_d, were predicted to have mutations in the target site G1. Subsequently, sequencing analysis revealed that all four plants held heterozygous mutated alleles with the identical $1 \mathrm{bp}$ insertion in the G1 target site (Figure 2A; chromatograms are shown in Supplementary Figure S2), even though they were regenerated from different calli. Because the CDS of PPR2 was predicted on the reverse strand of the G1 target site, we investigated the number of PPR motifs using TPRPred (https://toolkit.tuebingen.mpg.de/tools/ tprpred; Supplementary Figure S3). The PPR2 gene of CWA contained 13 PPR motives, whereas a premature stop codon was created in the 4 th PPR motif in the 
A
WT
$-2,444$
$-2,425$
CWA G1 a TGCAACCTGACTT GCAAAGACCAAACAGAATTT
CWA_G1_a TGCAACCT GACTTtGCAAAGACCAAACAGAATTT
$\begin{array}{ll}\text { CWA_G1_b } & \text { TGCAACCTGACTT GCAAAGACCAAACAGAATTT } \\ \text { TGCAACCT GACTTtGCAAAGACCAAACAGAATTT }\end{array}$
$\begin{array}{ll}\text { CWA_G1_c } & \text { TGCAACCTGACTT GCAAAGACCAAACAGAATTT } \\ \text { TGCAACCTGACTTtGCAAAGACCAAACAGAATTT }\end{array}$
CWA_G1_d $\begin{aligned} & \text { TGCAACCTGACTT GCAAAGACCAAACAGAATTT } \\ & \text { TGCAACCTGACTTtGCAAAGACCAACAGAATTT }\end{aligned}$

\section{B}

\begin{tabular}{|c|c|c|}
\hline & motif 4 & \\
\hline & TTVDRLIKKAVSGSNFELALKVFDLMLSLCYFPTL & \\
\hline & & \\
\hline & TTVDRLIKKAVSGSNFELALKVFDLMLSLCY & \\
\hline & motif 5 & \\
\hline VA_WT & $\overline{P I A N A I V S I L L K S G S A E I A Y E V F M V L V N R K F V P D V ~}$ & \\
\hline WA_G1 & PIANAIVSILLKSGSAEIAYEVFMVLVNRKFVPDV & \\
\hline & motif 6 & \\
\hline & YMYNQILFGLCKSGCSNKALTLFC & \\
\hline & AYYO I I I CKYPIS & \\
\hline
\end{tabular}

Figure 2. Mutated nucleotide and predicted amino acid sequences of genome-edited CWA plant, CWA_G1. (A) Nucleotide sequences around the G1 target sites in CWA_G1 plants in the $\mathrm{T}_{0}$ generation. The G1 target sequence is underlined and red lowercase letter indicates the insertion. The PAM sequence is shown in blue. WT, untransformed wild-type. Nucleotide positions from the start codon of $R M S$ are indicated. (B) The comparison of amino acid sequences of PPR2 among untransformed CWA_WT, CWR _WT, and CWA_G1. PPR motif is indicated. * Indicates a premature stop codon.

WT

$-931$ AAACCCGTCACCATCGGTGGCCGTCCCCCCTGGGCCTCCGCCC

CWA_G2_a AAA $\ldots \ldots-\ldots, \ldots$ nt $\ldots \ldots$ AAA - - -

Figure 3. Mutated nucleotide sequences of the genome-edited CWA plant, CWA_G2. The nucleotide sequences around the G2 target site in CWA_G2_a in the $\mathrm{T}_{0}$ generation. The G2 target sequence in underlined and dashes and red letters indicate deletion and the number of deleted nucleotides, respectively. The PAM sequence is shown in blue. WT, untransformed wild-type. Nucleotide positions from the start codon of $R M S$ are indicated.

fertility restorer line CWR because of the SNP. The $1 \mathrm{bp}$ insertion in the G1 target site created a premature stop codon in the 6th PPR motif in the mutated allele of CWA_G1_a to CWA_G1_d (Figure 2B).

We also analyzed transgenic plants with the G2 construct and obtained one plant containing a homozygous mutated allele with a $37 \mathrm{bp}$ deletion on the G2 target site, which we named CWA_G2_a (Figure 3; chromatograms are shown in Supplementary Figure S4).

\section{Seed setting rates of CWA_G1 and CWA_G2 plants}

Seed setting rates of CWA, CWR, and the transgenic
A

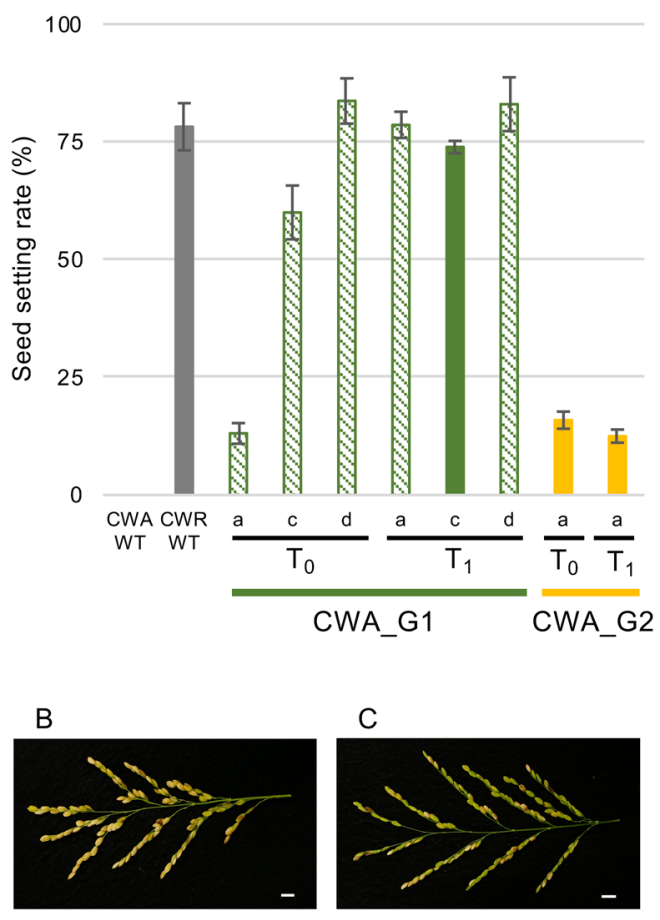

Figure 4. Fertility restoration in genome-edited CWA plants, CWA_ G1 and CWA_G2. (A) Seed setting rates of untransformed CWA WT, CWR_WT and CWA_G1 and CWA_G2 plants in the $\mathrm{T}_{0}$ and $\mathrm{T}_{1}$ generations. An error bar indicates SE obtained from the values of three to five panicles per plant. Striped and solid bars indicate that plants carried the heterozygous and homozygous mutated alleles, respectively. The $\mathrm{T}_{0}$ plant of CWA_G1_a was grown in a greenhouse, while all other plants were grown in a biotron. (B and C) Panicles of the CWA_G1_a and CWA_G2_a in the $\mathrm{T}_{1}$ generation. Scale bar $=1.0 \mathrm{~cm}$.

plants with the mutated alleles (CWA_G1_a, CWA_ G1_c, CWA_G1_d, and CWA_G2_a) were examined (Figure 4A). All the transgenic plants exhibited restored fertility and set seeds upon self-pollination. The resulting $\mathrm{T}_{1}$ seeds germinated normally and the $\mathrm{T}_{1}$ plants showed normal vegetative growth without any morphological defects. The $T_{0}$ plant of CWA_G1_a was grown in a greenhouse, which might cause the lower seed setting rate of $13.1 \%$, while all other plants were grown in a biotron for further investigation of the seed setting rates. The highest seed setting rates among the CWA_G1 in each generation was $83.7 \%$ in $\mathrm{T}_{0}$ and $83.0 \%$ in $\mathrm{T}_{1}$, and the values were almost identical to that of CWR. Likewise, in CWA_G2_a, the values were $15.9 \%$ in $\mathrm{T}_{0}$ and $12.5 \%$ in $\mathrm{T}_{1}$. The fertility of CWA_G2 was relatively lower compared to those of CWA_G1 (Figure 4B, C). Because the wild-type CWA is a completely sterile CMS line with no seed setting, these results indicated that we successfully created two new alleles that restored CW-CMS.

Seed setting rates were also examined in three plants in the $\mathrm{T}_{2}$ generation derived from CWA_G1_a. They were $79.3 \%$ and $62.4 \%$ for the plants with the 


\begin{tabular}{|l|l|l|l|}
\hline CWA G1_a T2 & \multicolumn{1}{|c|}{$\# 1$} & \multicolumn{1}{|c|}{$\# 2$} & \multicolumn{1}{|c|}{$\# 3$} \\
\hline $\begin{array}{l}\text { Zygosity of mutated } \\
\text { allele }\end{array}$ & Hetero & Homo & Homo \\
\hline $\begin{array}{l}\text { Seed setting rates } \\
\text { (\%; Mean } \pm \text { SE) }\end{array}$ & $\begin{array}{l}63.0 \pm \\
9.8\end{array}$ & $\begin{array}{l}79.3 \pm \\
1.9\end{array}$ & $\begin{array}{l}62.4 \pm \\
5.0\end{array}$ \\
\hline
\end{tabular}

Detection of HPT gene

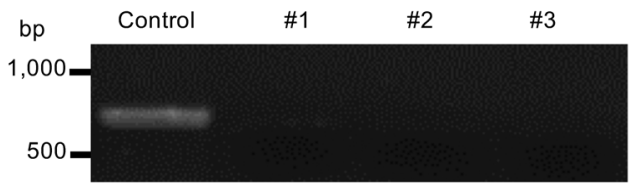

Figure 5. Fertility restoration in null-segregants of the genome-edited plants. Seed setting rates (\%; Mean \pm SE) of CWA_G1_a plants carrying homozygous or heterozygous mutated alleles in the $\mathrm{T}_{2}$ generation and PCR detection of the HPT gene for verification of the absence of the introduced sgRNA/Cas9-expression construct.

homozygous mutated allele, and $63.0 \%$ for the plant with the heterozygous mutated allele (Figure 5). In the two homozygous plants, the nucleotide sequence at the G1 target sites was confirmed to retain the insertion of thymine identical to that of the $\mathrm{T}_{0}$ generation by direct sequencing (Supplementary Figure S5). As shown in Figure 5, the foreign gene, HPT of the sgRNA/Cas9expression construct, was not detected by PCR analysis in these two plants carrying the homozygous mutated allele and one plant carrying heterozygous mutated alleles in the $\mathrm{T}_{2}$ generation; thus, they were nullsegregants for the introduced gene.

\section{RMS and PPR2 expression analysis of CWA_G1 and $G 2$}

To investigate the relationship between gene expression of PPR2 and RMS and the fertility recovery of the genome-edited plants of CWA, we analyzed these transcripts in mature anthers of CWA, CWR, and CWA G1_c, and CWA_G2_a plants with the homozygous mutated alleles by quantitative real-time RT-PCR (qRTPCR; Figure 6). For PPR2 expression, no significant differences were observed among the plants. However, the expression level of RMS in CWA_G1_c was significantly decreased compared to that of CWA and was identical to that of CWR. The expression level of RMS in CWA_G2_a was also decreased compared to untransformed CWA_WT, although the difference was not statistically significant. These results suggested that the G2 target site was less important than the G1 target site for decreasing RMS expression. Because the mutation at the G2 site did not affect CDS of PPR2 and the RNA level of PPR2 was not changed in CWA_G2_a, we could rule out the possibility that $P P R 2$ was involved in fertility restoration, and conclude the reduced RMS expression was responsible for fertility restoration of CW-CMS.

\section{Disruption of RMS CDS}

To investigate whether disruption of the RMS CDS

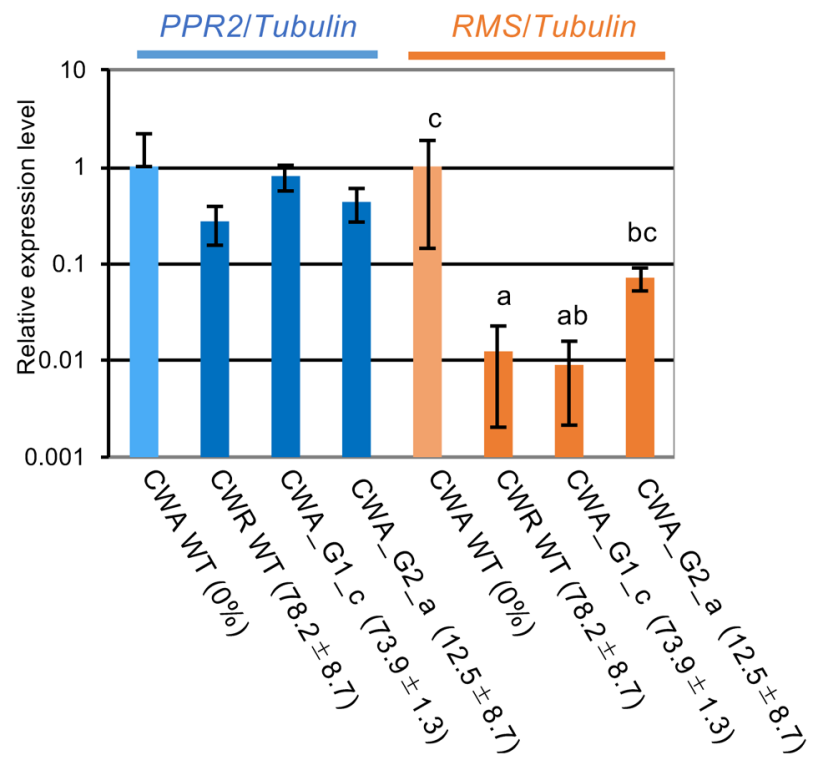

Figure 6. Relative expression level of RMS and PPR2. qRT-PCR analysis of relative PPR2 and RMS mRNA levels in the mature anthers of the $\mathrm{T}_{1}$ transgenic plants and CWA and CWR_WT plants. The transcription level of tubulin was used as an internal control of gene expression. Error bars indicate standard deviation obtained from the values of four biological replicates. The different alphabets above the bars represent significant differences among the samples at $p<0.05$ by Tukey's test. The numbers under the name of plants represent seed setting rates (\%; mean $\pm \mathrm{SE}$ ) of plants, which were used for qRT-PCR analysis.

induced fertility restoration, we introduced the sgRNA/ Cas9-expression vectors, G6 and G7, whose target sites were within the CDS of RMS, into CWA and T65. Fifteen independent CWA transgenic plants and five independent T65 transgenic plants (T65_G6 and T65_ G7) were obtained for each vector. Although we screened mutations at the target regions in these transgenic plants for the $\mathrm{T}_{0}$ generation, mutations were not detected by the IndelCheck ${ }^{\mathrm{TM}}$ CRISPR/TALEN Insertion or Deletion Detection System. We suspected that even if there were no edited alleles in the $T_{0}$ plants, we might obtain edited alleles in the next generation. Because those of $\mathrm{T}_{0}$ plants of the transgenic CWA_G6 and CWA_G7 were sterile, we made a cross between CWA_G6 (female) and the pollen of fertile T65_G6, and between CWA_ G7 (female) with the pollen of fertile T65_G7. Finally, we obtained the $T_{1}$ generation that harbored G6 or G7 sgRNA/Cas9-expression constructs in the nucleus and the CW mitochondria in the cytoplasm. Then, we screened these $\mathrm{T}_{1}$ plants using the IndelCheck ${ }^{\mathrm{TM}}$ CRISPR/ TALEN Insertion or Deletion Detection System and found mutations in six of 21 plants for the G6 sites and three out of 27 plants for the G7 sites. All these nine plants were shown to possess HPT in the sgRNA/ Cas9-expression vector (Supplementary Figure S6). Deletions and insertions, which caused knockouts of the RMS allele, were found by nucleotide sequencing of three independent clones of PCR-amplified products in 
A

\begin{tabular}{|c|c|}
\hline WT & $\begin{array}{cc}+105 & +124 \\
\text { CAGCCACGACAGCACATGGGCAGGGATG }\end{array}$ \\
\hline CWA_G6_a & $\begin{array}{l}\text { CAGCCACGACAGCACATGGGCAGGGATG } \\
\text { CAGCCACGg--- CATGGGCAGGGATG }\end{array}$ \\
\hline CWA_G6_b & $\begin{array}{l}\text { CAGCCACGACAGCACATGGGCAGGGATG } \\
\text { CAGCCACGACA-- } \underline{\text { TGGGCAGGGATG }}\end{array}$ \\
\hline CWA_G6_C & $\begin{array}{l}\text { CAGCCACGACAGCACATGGGCAGGGATG } \\
\text { CAGCCACGAtAGCACATGGGCAGGGATG } \\
\text { CAGCCACGA-AGCACATGGGCAGGGATG }\end{array}$ \\
\hline CWA_G6_d & $\begin{array}{l}\text { CAGCCACGACAGCACATGGGCAGGGATG } \\
\text { CAGCCACGAC-GCACATGGGCAGGGATG }\end{array}$ \\
\hline CWA_G6_e & $\begin{array}{l}\text { CAGCCACGACAGCACATGGGCAGGGATG } \\
\text { CAGCCACGtCAGCACATGGGCAGGGATG } \\
\text { CAGCCACGA-- ACATGGGCAGGGATG } \\
\text { CAGCCACGA-AGCACATGGGCAGGGATG }\end{array}$ \\
\hline CWA_G6_f & $\begin{array}{l}\text { CAGCCACGACAGCACATGGGCAGGGATG } \\
\text { CAGCCACGA-AGCACATGGGCAGGGATG }\end{array}$ \\
\hline
\end{tabular}

B WT

CWA_G7_a

CWA_G7_b

CWA_G7_C

+96
AAGATGCAGCCACGACAGCACATGGGCAG

AAGATGCAGCCACGACAGCA CATGGGCAG AAGATGCAGCCACGACAGCAgCATGGGCAG

AAGATGCAGCCACGACAGCACATGGGCAG AAGATGCAGCCACGACA----IGGGCAG

AAGATGCAGCCACGACAGCACATGGGCAG

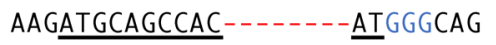
AAGATGCAGCCACGACAGCAgCATGGGCAG AAGATGCAGCCACGACAGCATCATGGGCAG

Figure 7. Mutated nucleotide sequences of genome-edited CWA plants, CWA_G6 (A) and CWA_G7 (B). The nucleotide changes (dashes in red for deletion, lowercase letters in green for substitutions and purple for insertions) are indicated in the sequences. The G6 and G7 target sequences are underlined. The PAM sequence is shown in blue. WT, untransformed wild-type. Nucleotide positions from the start codon of RMS are indicated.

CWA_G6_a, CWA_G6_b, CWA_G6_c, CWA_G6_d, CWA_G6_e, CWA_G6_f, CWA_G7_a, CWA_G7_b, and CWA_G7_c (Figure 7; Chromatograms are shown in Supplementary Figure S7). CWA_G6_c, CWA_G6_e, and CWA_G7_c were likely to be chimeric mutants.

Seed setting rates were investigated for these nine plants. None set any seeds. The results are in contrast to our expectation that the plants with a heterozygous knockout allele might set seeds because $R f 17$ acts gametophytically in pollen and allows the plant with Rf17rf17 to set seeds normally. These results indicated that knockout of RMS does not restore fertility of CW-CMS.

\section{Discussion}

Fertility was restored by a mutation in the promoter of RMS

In this study, we created two new alleles that restored CW-CMS. As shown in Figure 4, fertility was fully restored in CWA_G1 and partially restored in CWA_G2 plants. The G1 target site exists at $-2,425$ to $-2,444 \mathrm{bp}$ upstream from the RMS start codon, which was near the SNP site at $-2,286$ bp in the original fertility restorer line CWR (Figures 1-3). It is assumed that the G1 target site was more important for reducing RMS expression and leading to fertility restoration than the G2 target site at -912 to -931 bp (Figure 6). Further study of additional mutants is needed to delimit the cis-element responsible for RMS expression. Examples in which regulatory sequences located distant to the CDS have also been reported in other genes. The expression of QTL of seed shattering in chromosome 1 (qSH1) and FLOWERING LOCUS $T$ are regulated by $12 \mathrm{~kb}$ and $5.7 \mathrm{~kb}$ upstream of their start codon (Konishi et al. 2006; Liu et al. 2014). Considering the analysis of the CWA_G2 plant, in which the target site was located in the promoter of $R M S$ outside of the CDS of PPR2, we concluded that PPR2 is not linked to CW-CMS. This is in contrast to most studies of $R F$ genes that encode $P P R$ proteins.

A premature stop codon was created in PPR 2 of CWR_WT and CWA_G1 plants, which indicated that these plants lost the function of PPR2 protein. Because no defective phenotypes were observed in these plants, the function of $P P R 2$ is still unknown.

The seed setting rates of the CWA_G1 plants were higher than those of the RNAi-mediated transgenic plants with slightly reduced expression of RMS in our previous study (Fujii and Toriyama 2009). The current study clearly demonstrated that the reduced expression allele is responsible for the fertility restoration of CW-CMS.

\section{Hypotheses why fertility was not restored in RMS knockout lines}

We obtained nine transgenic lines which harbor RMS knockout alleles. We expected, at the beginning of this study, that they would restore fertility. All of them, however, showed no restoration of fertility. We would rule out the possibility that it was caused by an offtarget effect, because it was unreasonable to think that both of the G6 and G7 mutants suffered from the same off-target effect that cause sterility. The reason why CWCMS was not restored in the RMS knockout lines is still unknown. Because the mRNA expression level of RMS in mature anthers of a normal fertile cultivar T65 has been reported to be approximately half that of CWA (Fujii and Toriyama 2009), it is possible that the knockout RMS allele might be lethal to pollen development. 
The RMS encodes a protein 178 aa long, and contain a 139th to 156th aa segment similar to a part of the acylcarrier protein synthase (ACPS) domain. It is known that ACPS is directly related to the elongation of 18:0 ACP (Nishida 2004). Thus, we speculated that RMS has another function related to ACPS and involved in pollen development directly or indirectly. Regarding ACPS in rice, knockout of OsACOS12, which encodes a rice fatty acyl-CoA synthetase, has been reported to cause pollen abortion (Yang et al. 2017; Zou et al. 2017). In tobacco, the $\beta$-Ketoacyl-[acyl carrier protein] synthase I (KASI) mutant has been reported to cause abnormal development of embryos and differentiation of chloroplasts and reduce seed setting rates ( $\mathrm{Wu}$ and Xue 2010). In addition, there were several reports that revealed the knockout of genes related to acyl-carrier protein caused male sterility, including Defective Pollen Wall in rice that encodes a fatty acyl-carrier protein reductase (Shi et al. 2011), Defective Pollen Wall 2 in rice that encodes an acyl transferase (Xu et al. 2017), and Male Sterile2 in Arabidopsis that encodes a fatty acylcarrier protein reductase (Chen et al. 2011). Recently, an $R F$ gene, which has a function other than fertility restoration in CMS, has been reported. Rf4 in maize, which has been cloned as an $R F$ gene for C-type CMS, has been reported to have another function that regulates the development and specification of the tapetum (Jaqueth et al. 2020; Nan et al. 2017). Considering the above reports, we suggest that RMS might not only be related to CW-CMS but also have an essential function for pollen development.

\section{Creation of a new artificial restorer line}

CMS is an agronomically important trait often used for $\mathrm{F}_{1}$ hybrid breeding, as most commercial hybrid crops have been developed based on a three-line system, namely a CMS line, a maintainer line, and a restorer line. $\mathrm{F}_{1}$ hybrid seeds are produced by crossing a CMS line and a restorer line. Because of hybrid vigor, the $\mathrm{F}_{1}$ hybrid rice grows stronger and results in 15 to $30 \%$ higher yields than conventional inbred lines (Huang et al. 2014). CWCMS has been demonstrated to be useful for producing CMS lines of many elite Indica Group rice cultivars (Toriyama and Kazama 2016; Toriyama et al. 2019).

We obtained the null-segregant from the CWA_G1_a in the $\mathrm{T}_{2}$ generation, which did not harbor a foreign gene, but still carried mutated alleles homozygously and exhibited as high as a $79.3 \%$ seed setting rate (Figure 5). The seed setting rates were the same as that of the original CWR and were high enough to use as a new restorer line for practical breeding. We will be able to easily produce artificial restorer lines on demand by introducing the G1 sgRNA/Cas9 expression vector. The creation of artificial restorer lines has not yet been reported for any other crops or vegetables.

\section{Acknowledgements}

We thank Drs. Masaki Endo and Seiichi Toki (National Institute of Agrobiological Sciences, Tsukuba, Japan) for providing Cas9 plant expression vector (pZH_gYSA_MMCas9) and sgRNA expression vector (pU6gRNA). This study was partly supported by KAKENHI $17 \mathrm{H} 03744$.

\section{References}

Akagi H, Nakamura A, Yokozeki-Misono Y, Inagaki A, Takahashi H, Mori K, Fujimura T (2004) Positional cloning of the rice $R f-1$ gene, a restorer of BT-type cytoplasmic male sterility that encodes a mitochondria-targeting PPR protein. Theor Appl Genet 108: 1449-1457

Bohra A, Jha UC, Adhimoolam P, Bisht D, Singh NP (2016) Cytoplasmic male sterility (CMS) in hybrid breeding in field crops. Plant Cell Rep 35: 967-993

Chen W, Yu XH, Zhang K, Shi J, De Oliveira S, Schreiber L, Shanklin J, Zhang D (2011) Male Sterile2 encodes a plastidlocalized fatty acyl carrier protein reductase required for pollen exine development in Arabidopsis. Plant Physiol 157: 842-853

Cong L, Ran FA, Cox D, Lin S, Barretto R, Habib N, Hsu PD, Wu X, Jiang W, Marraffini LA, et al. (2013) Multiplex genome engineering using CRISPR/Cas systems. Science 339: 819-823

Fujii S, Kazama T, Yamada M, Toriyama K (2010) Discovery of global genomic re-organization based on comparison of two newly sequenced rice mitochondrial genomes with cytoplasmic male sterility-related genes. BMC Genomics 11: 209

Fujii S, Toriyama K (2005) Molecular mapping of the fertility restorer gene for $m s$ - $C W$-type cytoplasmic male sterility of rice. Theor Appl Genet 111: 696-701

Fujii S, Toriyama K (2009) Suppressed expression of RETROGRADE-REGULATED MALE STERILITY restores pollen fertility in cytoplasmic male sterile rice plants. Proc Natl Acad Sci USA 106: 9513-9518

Hanson MR, Bentolila S (2004) Interactions of mitochondrial and nuclear genes that affect male gametophyte development. Plant Cell 16 (suppl): S154-S169

Hothorn T, Bretz F, Westfall P (2008) Simultaneous inference in general parametric models. Biom J 50: 346-363

Hu J, Wang K, Huang W, Liu G, Gao Y, Wang J, Huang Q, Ji Y, Qin X, Wan L, et al. (2012) The rice pentatricopeptide repeat protein RF5 restores fertility in Hong-Lian cytoplasmic male-sterile lines via a complex with the glycine-rich protein GRP162. Plant Cell 24: $109-122$

Huang JZ, Zhi-Guo E, Zhang HL, Shu QY (2014) Workable male sterility systems for hybrid rice: Genetics, biochemistry, molecular biology, and utilization. Rice (NY) 7: 13

Huang W, Yu C, Hu J, Wang L, Dan Z, Zhou W, He C, Zeng Y, Yao G, Qi J, et al. (2015) Pentatricopeptide-repeat family protein RF6 functions with hexokinase 6 to rescue rice cytoplasmic male sterility. Proc Natl Acad Sci USA 112: 14984-14989

Itabashi E, Iwata N, Fujii S, Kazama T, Toriyama K (2011) The fertility restorer gene, $R f 2$, for Lead Rice-type cytoplasmic male sterility of rice encodes a mitochondrial glycine-rich protein. Plant J 65: 359-367

Jaqueth JS, Hou Z, Zheng P, Ren R, Nagel BA, Cutter G, Niu X, Vollbrecht E, Greene TW, Kumpatla SP (2020) Fertility restoration of maize CMS-C altered by a single amino acid substitution within the Rf4 bHLH transcription factor. Plant $J$ 101: 101-111 
Jiang W, Bikard D, Cox D, Zhang F, Marraffini LA (2013) RNAguided editing of bacterial genomes using CRISPR-Cas systems. Nat Biotechnol 31: 233-239

Kazama T, Nakamura T, Watanabe M, Sugita M, Toriyama K (2008) Suppression mechanism of mitochondrial ORF79 accumulation by Rf1 protein in BT-type cytoplasmic male sterile rice. Plant $J$ 55: 619-628

Kazama T, Toriyama K (2003) A pentatricopeptide repeatcontaining gene that promotes the processing of aberrant atp 6 RNA of cytoplasmic male-sterile rice. FEBS Lett 544: 99-102

Kazama T, Toriyama K (2014) A fertility restorer gene, $R f 4$, widely used for hybrid rice breeding encodes a pentatricopeptide repeat protein. Rice (NY) 7: 28

Kitazaki K, Arakawa T, Matsunaga M, Yui-Kurino R, Matsuhira H, Mikami T, Kubo T (2015) Post-translational mechanisms are associated with fertility restoration of cytoplasmic male sterility in sugar beet (Beta vulgaris). Plant J 83: 290-299

Koizuka N, Imai R, Fujimoto H, Hayakawa T, Kimura Y, KohnoMurase J, Sakai T, Kawasaki S, Imamura J (2003) Genetic characterization of a pentatricopeptide repeat protein gene, orf687, that restores fertility in the cytoplasmic male-sterile Kosena radish. Plant J 34: 407-415

Komori T, Ohta S, Murai N, Takakura Y, Kuraya Y, Suzuki S, Hiei Y, Imaseki H, Nitta N (2004) Map-based cloning of a fertility restorer gene, $R f-1$, in rice (Oryza sativa L). Plant J 37: 315-325

Konishi S, Izawa T, Lin SY, Ebana K, Fukuta Y, Sasaki T, Yano M (2006) An SNP caused loss of seed shattering during rice domestication. Science 312: 1392-1396

Li JF, Norville JE, Aach J, McCormack M, Zhang D, Bush J, Church GM, Sheen J (2013) Multiplex and homologous recombinationmediated genome editing in Arabidopsis and Nicotiana benthamiana using guide RNA and Cas9. Nat Biotechnol 31: 688-691

Liu F, Schnable PS (2002) Functional specialization of maize mitochondrial aldehyde dehydrogenases. Plant Physiol 130: $1657-1674$

Liu L, Adrian J, Pankin A, Hu J, Dong X, Von Korff M, Turck F (2014) Induced and natural variation of promoter length modulates the photoperiodic response of FLOWERING LOCUS T. Nat Commun 5: 4558

Mikami M, Toki S, Endo M (2015) Comparison of CRISPR/Cas9 expression constructs for efficient targeted mutagenesis in rice. Plant Mol Biol 88: 561-572

Nan GL, Zhai J, Arikit S, Morrow D, Fernandes J, Mai L, Nguyen N, Meyers BC, Walbot V (2017) MS23, a master basic helix-loophelix factor, regulates the specification and development of the tapetum in maize. Development 144: 163-172

Nekrasov V, Staskawicz B, Weigel D, Jones JD, Kamoun S (2013) Targeted mutagenesis in the model plant Nicotiana benthamiana using Cas9 RNA-guided endonuclease. Nat Biotechnol 31: 691-693

Nishida I (2004) Plastid matabolic pathways for fatty acid metabolism. In: Daniell H, Chase C (eds) Molecular Biology and
Biotechnology of Plant Organelles. Springer, The Netherlands, pp 543-564

Ohnishi T, Yoshino M, Yamakawa H, Kinoshita T (2011) The biotron breeding system: A rapid and reliable procedure for genetic studies and breeding in rice. Plant Cell Physiol 52: $1249-1257$

Schmitz-Linneweber C, Small I (2008) Pentatricopeptide repeat proteins: A socket set for organelle gene expression. Trends Plant Sci 13: 663-670

Shan Q, Wang Y, Li J, Zhang Y, Chen K, Liang Z, Zhang K, Liu J, Xi JJ, Qiu JL, et al. (2013) Targeted genome modification of crop plants using a CRISPR-Cas system. Nat Biotechnol 31: 686-688

Shi J, Tan H, Yu XH, Liu Y, Liang W, Ranathunge K, Franke RB, Schreiber L, Wang Y, Kai G, et al. (2011) Defective pollen wall is required for anther and microspore development in rice and encodes a fatty acyl carrier protein reductase. Plant Cell 23: 2225-2246

Tang H, Luo D, Zhou D, Zhang Q, Tian D, Zheng X, Chen L, Liu YG (2014) The rice restorer Rf4 for wild-abortive cytoplasmic male sterility encodes a mitochondrial-localized PPR protein that functions in reduction of WA352 transcripts. Mol Plant 7: 1497-1500

Toriyama K, Kazama T (2016) Development of cytoplasmic male sterile IR24 and IR64 using CW-CMS/Rf17 system. Rice (NY) 9: 22

Toriyama K, Kazama T, Sato T, Fukuta Y, Oka M (2019) Development of cytoplasmic male sterile lines and restorer lines of various elite Indica Group rice cultivars using CW-CMS/Rf17 system. Rice (NY) 12: 73

Uyttewaal M, Arnal N, Quadrado M, Martin-Canadell A, Vrielynck N, Hiard S, Gherbi H, Bendahmane A, Budar F, Mireau H (2008) Characterization of Raphanus sativus pentatricopeptide repeat proteins encoded by the fertility restorer locus for Ogura cytoplasmic male sterility. Plant Cell 20: 3331-3345

Wang Z, Zou Y, Li X, Zhang Q, Chen L, Wu H, Su D, Chen Y, Guo J, Luo D, et al. (2006) Cytoplasmic male sterility of rice with boro II cytoplasm is caused by a cytotoxic peptide and is restored by two related PPR motif genes via distinct modes of mRNA silencing. Plant Cell 18: 676-687

Wu GZ, Xue HW (2010) Arabidopsis $\beta$-ketoacyl-[acyl carrier protein] synthase I is crucial for fatty acid synthesis and plays a role in chloroplast division and embryo development. Plant Cell 22: 3726-3744

Xu D, Shi J, Rautengarten C, Yang L, Qian X, Uzair M, Zhu L, Luo Q, An G, Waßmann F, et al. (2017) Defective Pollen Wall 2 (DPW2) encodes an acyl transferase required for rice pollen development. Plant Physiol 173: 240-255

Yang X, Liang W, Chen M, Zhang D, Zhao X, Shi J (2017) Rice fatty acyl-CoA synthetase OsACOS12 is required for tapetum programmed cell death and male fertility. Planta 246: 105-122

Zou T, He Z, Qu L, Liu M, Zeng J, Liang Y, Wang T, Chen D, Xiao Q, Zhu J, et al. (2017) Knockout of OsACOS12 caused male sterility in rice. Mol Breeding 37: 126 